\title{
Bias adjustment in multilevel meta-analysis of standardized single-case experimental data
}

Laleh Jamshidia ${ }^{a}$ Lies Declercqa ${ }^{\mathrm{a}}$, Belén Fernández-Castilla ${ }^{\mathrm{a}}$, John M. Ferron ${ }^{\mathrm{b}}$, Mariola Moeyaert $^{\mathrm{c}}$, S. Natasha Beretvas ${ }^{\mathrm{d}}$, and Wim Van den Noortgate ${ }^{\mathrm{a}}$

${ }^{a}$ Faculty of Psychology and Educational Sciences \& imec-itec, KU Leuven, University of Leuven,

$$
\text { Belgium; }
$$

${ }^{\mathrm{b}}$ Department of Educational Measurement and Research, University of South Florida, Tampa, Florida, USA;

${ }^{\mathrm{c}}$ Department of Educational and Counseling Psychology, University at Albany (State University of New York);

${ }^{\mathrm{d}}$ Department of Educational Psychology, University of Texas, Austin

*Correspondence concerning this article should be addressed to Laleh Jamshidi, Faculty of Psychology and Educational Sciences, KU Leuven, Etienne Sabbelaan 51, room: 01.33, 8500 Kortrijk, Belgium, E-mail: laleh.jamshidi@kuleuven.be, Phone: +32497360163 . 


\section{Abstract}

In recent years, multilevel meta-analysis has become an increasingly popular way to combine the results of single-case experimental designs (SCEDs). Previous research found bias in the estimate of the overall fixed effects and variance components using multilevel meta-analyses of standardized single-case data. Therefore, we propose and evaluate two adjustments in an attempt to reduce the bias and improve the statistical properties of the parameter estimates, more specifically the use of Hedges' correction factor and constraining the variance at the first level. The results confirm the existence of bias when estimating an overall effect based on standardized data, as well as bias when estimating variance components. The relative bias decreases as the number of measurement occasions increases. The results indicate that the between-case variance and to a smaller degree the between-study variance is overestimated, especially when the number of measurement occasions per phase is only 10. In general, Hedges' bias correction procedure led to better estimations of both fixed effects and variance components. In contrast, constraining the level-1 variance hardly affected the results.

Keywords: Single-case experimental design (SCED), Multilevel meta-analysis, Bias correction 


\section{Introduction}

A single-case experimental design (SCED) is a kind of time-series design in which a case -a single participant or another unit such as a group or organization- is repeatedly measured over time on the outcome of interest. In such a design, the repeated measurements under at least two different conditions (i.e., prior and during the intervention) can be compared to assess the effectiveness of the intervention of interest (Horner et al., 2005; Kratochwill et al., 2010). An SCED may be an appropriate alternative for a group comparison design in situations where the researcher is interested in rare phenomena (e.g., studying people with specific disabilities or specific diseases for which multiple cases may not exist; Barlow, Nock, \& Hersen, 2009; Kratochwill et al., 2010; Onghena, 2005). This kind of design has other advantages including: this design allows the practitioners to implement SCEDs in their natural setting to decrease the gap between the research and practice (Morgan \& Morgan, 2001), each subject acts as its own control for the purpose of comparison (Kratochwill et al., 2010), and it allows repeated measurements at various time occasions, therefore the treatment effect would be evaluated with more than a single observation, allowing the inspection of the treatment effects over time (Morgan \& Morgan, 2001).

SCEDs have been implemented in different disciplines such as education, psychology, medicine and health sciences, and social work (Kratochwill \& Levin, 2014; Riley-Tillman \& Burns, 2009). There are many varieties of SCEDs, such as basic AB designs or withdrawal designs (e.g., $\mathrm{ABA}, \mathrm{ABAB}, \mathrm{BAB}, \mathrm{ABCB}$ designs), in which A represents a baseline phase and $\mathrm{B}$ and $\mathrm{C}$ represent a treatment phase, multiple-baseline designs, and alternating treatments designs (Barlow et al., 2009; Horner \& Odom, 2014) or combinations of these designs. Given that an issue of SCED findings is the limited generalizability, a possible solution to increase the credibility of the results is replicating the experiment across cases, behaviors, or settings (Barlow et al., 2009; Kratochwill \& Levin, 2014). As the use of SCEDs has substantially grown 
in different fields, applying meta-analytic approaches to statistically synthesize the results of multiple SCEDs is a way to obtain more reliable evidence-based conclusion about specific treatment(s)/ intervention(s).

Various methods have been proposed by different scholars to express the size of the effect in primary SCEDs including the use of non-overlap indices (Parker, Vannest, \& Davis, 2014), standardized mean differences proposed by Hedges, Pustejovsky, \& Shadish (2012, 2013) which is comparable to those used in between-cases designs, or regression methods (Allison \& Gorman, 1993). For quantitatively combining the results of multiple primary SCEDs within or over studies, several meta-analytic methods can be applied to provide an estimate of the overall effect size or to study factors that moderate the effect. Researchers can calculate a simple mean, median, or range of effect sizes, calculate a precision weighted average of effect sizes, apply regression analyses to the effect sizes, or do multilevel meta-analyses of effect sizes from the primary SCEDs (Hedges et al., 2013, 2012; Petit-Bois, Baek, Van den Noortgate, Beretvas, \& Ferron, 2016; Shadish, 2014). An alternative approach is to combine the results of raw data (that can typically be extracted from the graphs reported in SCED studies) in a multilevel analysis, taking into account the hierarchical structure of the data (Van den Noortgate \& Onghena, 2003b, 2008).

In the current study, we focus on the use of multilevel models for the meta-analysis of both raw data and effect sizes. An advantage of the use of multilevel models is that it does not only give information on the overall treatment effect, but also on how the size of the effect varies over cases and over studies. Previous studies have consistently found that the recovery of variance components for the higher levels of the multilevel model (e.g., between-case variance and between-study variance) is rather poor in the sense of bias, unless there are many observations per case (e.g., Hembry, Bunuan, Beretvas, Ferron, \& Van den Noortgate, 2015; Moeyaert, Ugille, Ferron, Beretvas, \& Van den Noortgate, 2013, 2014; Ugille, Moeyaert, 
Beretvas, Ferron, \& Van den Noortgate, 2012). When the dependent variables from multiple SCED studies are not measured on the same scale, meta-analysts require to standardize data before combining them through multilevel modeling. Results were found to be slightly better when combining standardized effect sizes using a three-level meta-analysis of effect sizes (Ugille et al., 2012) compared to combining standardized raw data directly using a three-level analysis (Moeyaert et al., 2013). Two differences between the meta-analyses of standardized effect sizes (Ugille et al. 2012, 2014) and the multilevel data analyses of standardized raw data (Moeyaert et al., 2013) study are that in the former the variance at the lowest level (this is the variance of the observed effect sizes around the population values) is constrained to the sampling variances estimated prior to the meta-analysis, and that a small-sample bias-correction factor proposed by Hedges (1981) is applied to the effect sizes. Given the slightly better performance that was observed when analyzing effect sizes rather than raw data, it is interesting to explore whether applying adjustments in standardized raw data could lead to less bias in fixed effects and variance component estimates, particularly when the number of measurement occasions is small. No previous study has compared the performance of these adjustments in synthesizing the SCED raw data versus the SCED effect sizes.

To this end, a simulation study was designed to assess the statistical properties of the estimates of the fixed effects and especially of the higher-level residuals' variance components in the meta-analysis of standardized raw data and standardized effect sizes from multiplebaseline designs (MBDs). In such designs, an $\mathrm{AB}$ phase design is applied simultaneously to multiple cases, behaviors, or settings and the intervention introduction is staggered across time (Barlow et al., 2009; Horner \& Odom, 2014). We focus on MBD studies because such design is the most frequently applied SCED (Shadish \& Sullivan, 2011). The purpose of the present study is to find whether and to what degree the results can be improved by implementing these 
adjustments, i.e., Hedges' bias correction and constraining the level-1 residual's variance to one.

\section{Multilevel Meta-Analysis of SCEDs}

Van den Noortgate and Onghena (2003a, 2003b, 2008) proposed to use (generalized) linear mixed modeling, also known as multilevel or hierarchical modeling to combine the data of SCEDs. A linear mixed model (LMM) can be used for data that have a hierarchical structure as is the case in a SCED meta-analytic dataset: in a dataset consisting of data from multiple SCED studies, measurements at different time points (level 1) are nested within cases (level 2), which in turn are nested within higher level units, the studies (level 3). A three-level model can be applied to estimate the average treatment effects but also the variability at each of three levels (i.e., within-case, between-case, and between-study variances).

Van den Noortgate and Onghena (2003a, 2003b, 2008) suggested using a hierarchical model in which individual measurements are regressed on a time indicator, $T$, a dummy coded variable indicating whether the observation is from the baseline or treatment phase, $D$, and the interaction between $T$ and $D$. In this way, researchers can easily obtain insight in the immediate effect of the intervention and the intervention effect on the trend. At the first level, we use:

$$
Y_{i j k}=\beta_{0 j k}+\beta_{1 j k} T_{i j k}+\beta_{2 j k} D_{i j k}+\beta_{3 j k} T_{i j k} D_{i j k}+e_{i j k} \text { with } e_{i j k} \sim N\left(0, \sigma_{e}^{2}\right)
$$

in which, $i$ stands for the measurement occasion $(i=0,1, \ldots, I), j$ for the case $(j=0,1, \ldots, J)$, and $k$ for the study $(k=0,1, \ldots K)$. The dependent variable, $Y_{i j k}$, is the $i^{\text {th }}$ measurement outcome of case $j$ in study $k$. The regressor $D$ is a phase dummy variable which equals 0 in the baseline phase and 1 in the treatment phase and $T$ is a time variable (e.g., the session number). The equation shows that in the baseline phase, where $D_{i j k}$ equals 0 , the expected score for the $j^{\text {th }}$ 
case in study $k$ equals $\beta_{0 j k}+\beta_{1 j k} T_{i j k}$, while in the treatment condition, in which $D_{i j k}$ equals 1 , the expected score is $\left(\beta_{0 j k}+\beta_{2 j k}\right)+\left(\beta_{1 j k}+\beta_{3 j k}\right) T_{i j k}$. Coefficients $\beta_{2 j k}$ and $\beta_{3 j k}$ therefore refer to the treatment effect on the intercept and on the time trend, respectively. When centering the time variable around the value at the start of the treatment phase, $\beta_{0 j k}$ indicates the expected baseline level at the start of the treatment condition, $\beta_{1 j k}$ refers to linear trend during baseline, $\beta_{2 j k}$ indicates the immediate treatment effect at the start of the treatment phase, and $\beta_{3 j k}$ refers to the effect of treatment on the time trend.

This equation extends the model proposed by Center, Skiba, and Casey (1985) by including additional indices $j$ and $k$, allowing us to use a single equation for all cases and studies at the same time. Note that all regression coefficients also have indices $j$ and $k$, meaning that these can vary over cases and studies. The variation of the regression coefficients over cases and over studies is described using following equations at the second and third level of the model. These equations model the case-specific and study-specific regression coefficients (i.e., $\beta^{\prime}$ s or $\theta^{\prime}$ s) as the sum of a study-specific mean or overall population mean (i.e., $\theta^{\prime}$ s or $\gamma^{\prime} s$ ) plus a case-specific random effect or study-specific random effect (i.e., $u^{\prime} \mathrm{s}$ or $v^{\prime} \mathrm{s}$ ):

$$
\begin{gathered}
\left\{\begin{array}{l}
\beta_{0 j k}=\theta_{00 k}+u_{0 j k} \\
\beta_{1 j k}=\theta_{10 k}+u_{1 j k} \\
\beta_{2 j k}=\theta_{20 k}+u_{2 j k} \\
\beta_{3 j k}=\theta_{30 k}+u_{3 j k}
\end{array} \text { with }\left[\begin{array}{l}
u_{0 j k} \\
u_{1 j k} \\
u_{2 j k} \\
u_{3 j k}
\end{array}\right] \sim N\left(0, \Sigma_{u}\right)\right. \\
\left\{\begin{array}{l}
\theta_{00 k}=\gamma_{000}+v_{00 k} \\
\theta_{10 k}=\gamma_{100}+v_{10 k} \\
\theta_{20 k}=\gamma_{200}+v_{20 k} \\
\theta_{30 k}=\gamma_{300}+v_{30 k}
\end{array} \text { with }\left[\begin{array}{l}
v_{00 k} \\
v_{10 k} \\
v_{20 k} \\
v_{30 k}
\end{array}\right] \sim N\left(0, \Sigma_{v}\right)\right.
\end{gathered}
$$


Residuals at each level are assumed to be multivariate normally distributed with zero means. Parameters of primary interest are typically the $\gamma^{\prime} s$, in multilevel literature called fixed effects, in this case referring to the mean regression coefficients across studies, as well as the (co)variation in the regression coefficients over cases or studies (i.e. the elements of $\Sigma_{u}$ and $\left.\Sigma_{v}\right)$, in multilevel literature called the variance components. This multilevel approach makes it possible to estimate the mean treatment effect, to separate sampling variation, between-case variation and between-study variation in the treatment effects, and to study the moderating effect of case and study characteristics. The flexibility of multilevel modelling allows for multiple extensions or adaptations, such as including more complex time trends, including predictors at any level to study their moderating effects, accounting for autocorrelation, the use of complex covariance structures and modeling discrete dependent variables.

Due to the possibility that the dependent variable is measured on different scales in a set of included SCEDs in a meta-analysis, standardization can be applied to allow the immediate comparison and combination of scores or effect sizes across studies. This approach can be used for either standardizing raw data or standardizing effect sizes. Afterwards, the standardized data from primary SCEDs can be aggregated through multilevel analysis. To standardize the data, Van den Noortgate and Onghena (2003b, 2008) proposed to perform an ordinary least squares (OLS) regression analysis of raw data for each case in order to estimate the residual within-case standard deviation $\left(\hat{\sigma}_{e j k}\right)$ and the regression coefficients $\left(b_{2 j k}\right.$ and $b_{3 j k}$, corresponding to unstandardized intervention's effects on the level and on the slope by fitting one-level linear regression model) from Equation 1.

The raw data are then standardized by dividing them by the estimated residual withincase standard deviation (Equation 4). The standardized raw data are subsequently synthesized in a three-level analysis (Equations 1 to 3).

$$
Y_{i j k}^{\prime}=\frac{Y_{i j k}}{\widehat{\sigma}_{e j k}}
$$


Alternatively, the case-specific intervention effect size estimates (i.e., immediate effect and effect on time trend, $b_{2 j k}$ and $b_{3 j k}$ ) can be standardized by dividing them by the estimated residual's standard deviation $\left(\hat{\sigma}_{e j k}\right)$. The standardized effect sizes $\left(b^{\prime}{ }_{2 j k}\right.$ and $\left.b^{\prime}{ }_{3 j k}\right)$ are then aggregated in two separate univariate three-level meta-analyses for each type of these coefficients:

$$
\begin{array}{ll}
{b^{\prime}{ }_{2 j k}=\beta_{2 j k}+r_{2 j k}} & r_{2 j k} \sim N\left(0, \sigma_{r_{2 j k}}^{2}\right) \\
\beta_{2 j k}=\theta_{20 k}+u_{2 j k} & u_{2 j k} \sim N\left(0, \sigma_{u_{2 j k}}^{2}\right) \\
\theta_{2 j k}=\gamma_{200}+v_{20 k} & v_{2 j k} \sim N\left(0, \sigma_{v_{20 k}}^{2}\right)
\end{array}
$$

and

$$
\begin{array}{ll}
b^{\prime}{ }_{3 j k}=\beta_{3 j k}+r_{3 j k} & r_{3 j k} \sim N\left(0, \sigma_{r_{3 j k}}^{2}\right) \\
\beta_{3 j k}=\theta_{30 k}+u_{3 j k} & u_{3 j k} \sim N\left(0, \sigma_{u_{3 j k}}^{2}\right) \\
\theta_{3 j k}=\gamma_{300}+v_{30 k} & v_{3 j k} \sim N\left(0, \sigma_{v_{30 k}}^{2}\right)
\end{array}
$$

The residual variances of the first level are the sampling variances of the respective regression coefficients that were already estimated in the OLS regression analyses (i.e., $\sigma_{r_{2 j k}}^{2}$ and $\sigma_{r_{3 j k}}^{2}$ ) and therefore can be considered as known in the meta-analysis, while other variance components are estimated in the multilevel meta-analysis. In each of these two univariate threelevel models, one fixed effect (i.e., $\gamma_{200}$ or $\gamma_{300}$ ) and two variance components (i.e., $\sigma_{u_{2 j k}}^{2}$ and $\sigma_{v_{20 k}}^{2}$, or $\sigma_{u_{3 j k}}^{2}$ and $\left.\sigma_{v_{30 k}}^{2}\right)$ are estimated. 


\section{Adapting the Standardized SCED Data Analyses}

As mentioned above, in a multilevel meta-analysis of effect sizes, the level-1 variance is considered as known and constrained to the estimate of the sampling variance (Hox \& de Leeuw, 2003). Also for analyzing standardized raw data, the variance at the lowest level can in principle be constrained, given that due to standardization this variance is expected to be one.

Second, Hedges (1981) proposed to reduce the small sample bias from standardized mean differences obtained for group-comparison designs by multiplying the estimate by a correction factor:

$$
g^{c}=g c(m), \text { with } c(m)=1-\frac{3}{4 m-1}
$$

in which $g^{c}$ stands for the corrected effect size and $c(m)$ indicates the correction factor with $m$ equal to the degrees of freedom, which equals the total sample size of the group-comparison study minus two.

The application of the small sample bias correction was proposed to be used in betweencases standardized mean difference (Hedges et al., 2012, 2013), but also for the standardized regression coefficients calculated for each case separately (Ugille et al., 2014). The standardized SCED effect sizes have the estimated regression coefficients $b_{2 j k}$ and $b_{3 j k}$ as a normally distributed numerator and the residual standard deviation as a chi-squared distributed denominator. Consequently, the standardized effect sizes are expected to follow a non-central $t$ distribution. Standardized raw data are standardized in the same way: every raw score is divided by the estimated residual standard deviation. Therefore, the small sample bias correction proposed by Hedges (1981) is also expected to improve the results of the analysis of raw standardized SCED data. Given that SCEDs often have small sample sizes, applying the correction could help to reduce the bias (Shadish, Hedges, Horner, \& Odom, 2015). 
For SCED data, the degrees of freedom, $m$, is equal to the number of measurement occasions, $I$, minus the number of predictors, $p$, in the regression model (Equation 1 ) minus one (i.e., $\mathrm{m}=I-p-1)$. According to Equation 1, the degrees of freedom equals $I-4$. The bias-corrected standardized raw data and standardized effect size are:

$$
\begin{aligned}
& \left(Y_{i j k}^{\prime}\right)^{c}=Y_{i j k}^{\prime}\left(1-\frac{3}{4 m-1}\right) \\
& \left(b_{j k}^{\prime}\right)^{c}=b_{j k}^{\prime}\left(1-\frac{3}{4 m-1}\right)
\end{aligned}
$$

When the bias correction factor is applied to the standardized regression coefficient estimates, it should also be applied to the standard error estimates (and therefore the sampling variance) associated with each of the coefficients:

$$
\left(\widehat{\sigma_{b^{\prime}}^{2}}\right)^{c}=\widehat{\sigma_{b^{\prime}}^{2}}\left(1-\frac{3}{4 m-1}\right)^{2}
$$

\section{Methods}

In order to examine the performance of both adjustment procedures in the recovery of the fixed effects and the higher-level residuals' variance components in the meta-analysis of standardized raw data and standardized effect sizes from MBDs, raw data were generated using Equations $1-3$.

The simulation parameters representing the fixed and random effects, the number of measurement occasions, the number cases, and number of cases were selected to be similar to the simulation conditions in Ugille et al. (2012) and Ugille et al. (2014), as follows. We simulated MBD data in which the total number of simulated measurement occasions within a subject was set to a small number ( $I=10$ or 20$)$. Only data with a small number of measurement occasions were simulated because based on the results of studies of Ugille et al. (2012) and 
Ugille et al. (2014), it was found that only for a small number of measurements, estimates of the between-study variance and especially the between-case variance were distorted. Two values for the number of subjects per study were evaluated ( $J=4$ or 7$)$. The number of simulated studies $(K)$ equaled 10 or 30 . Because the data were generated from MBDs, the timing of intervention introduction was staggered across subjects (Table 1 describes the start points of the intervention for each case), similar to what has been done in Ugille et al. (2012).

[Table 1 near here]

Overall baseline coefficients $\left(\gamma_{000}\right.$ and $\left.\gamma_{100}\right)$ were set to zero. The overall immediate treatment effect and the treatment effect on time trend $\left(\gamma_{200}\right.$ and $\gamma_{300}$, respectively) were set to 2 and 0.2 , respectively. The diagonal elements of the between-case variance matrix, $\Sigma_{u}=$ $\operatorname{diag}\left(\sigma_{u_{0}}^{2}, \sigma_{u_{1}}^{2}, \sigma_{u_{2}}^{2}, \sigma_{u_{3}}^{2}\right)$, and the between-study variance matrix, $\Sigma_{v}=\operatorname{diag}\left(\sigma_{v_{0}}^{2}, \sigma_{v_{1}}^{2}, \sigma_{v_{2}}^{2}, \sigma_{v_{3}}^{2}\right)$, were generated according to two sets of conditions with values: $0.5,0.05,0.5,0.05$ and 2, 0.2, 2, 0.2. The covariances at the case and study levels were set to zero. For each combination of conditions, 2000 datasets were simulated, resulting in total to 64,000 datasets $(2 \times 2 \times 2 \times 2 \times 2$ x 2000).

Standardized raw data and standardized effect sizes were analyzed in multiple ways, in order to study to what extent the adjustment procedures improve the statistical properties of the fixed effect and the variance components estimates. For standardized raw data, the restricted maximum likelihood multilevel meta-analysis without any adjustment was compared to the approach applying Hedges' bias correction factor and to the analysis in which the level-1 residual's variance is constrained to one. In meta-analysis of standardized effect sizes, the level1 variance is always considered as known (and constrained equal to the sampling variances that were estimated prior to the meta-analyses). Therefore, for standardized effect sizes, the default likelihood-based model was compared to a meta-analysis applying Hedges' bias correction for 
both effects on level and effects on trend. We chose to use the Satterthwaite method for estimating the degrees of freedom, because it can be expected to provide relatively accurate confidence intervals for the estimates of the treatment effects (Ferron, Bell, Hess, RendinaGobioff, \& Hibbard, 2009). The multilevel analyses of the simulated data were carried out using the restricted maximum likelihood procedure. Data were simulated and analyzed in SAS 9.4 (Fan, Felsovalyi, Sivo, \& Keenan, 2002; SAS Institute Inc., 2017).

In order to evaluate the performance of the different approaches on the quality of fixed effect estimates, we looked at the relative bias in the estimates, which is the deviation of the treatment effect estimates from the true values divided by the true values. Furthermore, the mean square error (MSE) of estimates, the relative bias in the estimated standard errors, and the coverage proportion of $95 \%$ confidence intervals for fixed effects were studied. For each condition, the coverage proportion for a $95 \%$ confidence interval estimates of the mean effects was calculated, this is the proportion of intervals that include the true population value. To evaluate the bias of the standard error estimates for the fixed effects, which are used for calculating the confidence intervals or testing the statistical significance of the treatment effects, we compared the median standard error estimate to the standard deviation of all obtained effect size estimates in a condition (because a standard error is by definition the standard deviation of the sampling distribution of the estimate). We used the median rather than the mean standard error to evaluate the bias, because the distribution of standard error estimates tends to be skewed.

Then for the variance components, we examined the relative bias of the variance component estimates from the true population values. These indices were then compared across the different analysis approaches. Based on previous research, we considered a relative bias less than .05 as acceptable for the fixed effect estimates, a bias of less than .10 acceptable for the variance component estimates (Hoogland \& Boomsma, 1998). 
In order to study which design factors have the largest impact on the parameter recovery, analyses of variance (ANOVAs) were conducted by applying the PROC GLM procedure in SAS 9.4 and only effects with eta squared $\left(\eta^{2}\right)$ higher than .01 are discussed. We chose this cutoff, that is considered by Cohen (1988) as a small effect, because we found that when this effect occurs, it is clearly visible in our results.. In these analyses, the dependent variables included the relative bias of the treatment effect estimates, MSE of the treatment effect estimates, the relative difference between the corresponding estimated standard error and standard deviation of the treatment effects, the coverage proportion of $95 \%$ confidence intervals for fixed effects, and the relative bias of the variance component estimates from the true population values.

\section{Results}

The average treatment effects estimates (i.e., the immediate treatment effects and the treatment effects on the time trend) and the relative bias of variance components (i.e., between-case variance and between-study variance) are presented in the following sections. Because the patterns for the immediate treatment effect and the effect on the time trend were very similar, as well as the results of analyzing raw data and effect size data, we focus on the results for the immediate effect estimated aggregating standardized raw data in the text. We provide the results of both standardized raw data and standardized effect sizes in the tables, giving the possibility to the readers to compare the results of both kinds of analyses. Only in the case of different patterns, also the findings for standardized effect sizes and/or the effects on the time trend are discussed. 


\section{Average Treatment Effect}

\section{Relative deviation and variance}

The relative bias of the estimates of the immediate treatment effect $\left(\gamma_{200}\right)$ across all conditions and models ranged from -0.008 for the $K=10, J=4, I=10, \sigma_{u_{2}}^{2}=2, \sigma_{v_{2}}^{2}=2$ condition up to 0.154 for $K=10, J=4, I=10, \sigma_{u_{2}}^{2}=2, \sigma_{v_{2}}^{2}=0.5$. According to the results of the ANOVA, the design factors that affected the relative bias were the underlying model $\left(\eta^{2}=.056\right)$ and the number of measurement occasions $\left(\eta^{2}=.027\right)$. Relative bias for $\gamma_{200}$ is the largest (and positive) for small measurement occasions $(I=10)$. For larger numbers of measurement occasions $(I=20)$, the relative bias of the estimates at most of the conditions is very close to the cutoff (Hoogland \& Boomsma, 1998), so the estimates can be considered as unbiased.

When Hedges' correction factor was applied, estimates were consistently found unbiased, whereas constraining the level-1 variance to one, did not affect the bias observed in the default procedure. For instance, when $K=10, J=4, I=10, \sigma_{u_{2}}^{2}=2, \sigma_{v_{2}}^{2}=2$, the estimated relative bias of the immediate effect equaled 0.140 in the default procedure and also when constraining the level-1 variance to one, and -0.008 with Hedges' correction approach. In the constrained level-1 variance procedure, as well as in the standard procedure, less bias was observed as the number of measurement occasions increased. For Hedges' correction approach, there were no significant differences among bias estimates as the number of measurement occasions increased, because the effect sizes were unbiased in all conditions. Because the number of measurement occasions $(I)$ is the only factor that moderates the bias, the results in Table 2 are presented according to the different levels of measurement occasions.

[Table 2 near here] 
Figure 1 illustrates the distribution of the relative deviations of the estimates from the true population values across different procedures. The figure confirms that for the unadjusted procedure and for the one that constrains the level-1 variance to zero, the relative bias (this is the mean relative deviation) is smaller when the number of measurements increases, whereas there is no bias when Hedges' correction factor is applied. Moreover, the standard deviation of the deviations decreases across all procedures as the number of measurement occasions increases.

\section{[Figure 1 near here]}

\section{$M S E$}

The results regarding the MSE provide information about the variation of the estimates around the true population value. The smaller the MSE, the more optimal the estimates. For our simulated data, the smaller MSE was found when the number of measurement occasions and the number of studies increased. For all approaches the underlying model $\left(\eta^{2}=.013\right)$, the number of measurement occasions $\left(\eta^{2}=.021\right)$, the number of studies $\left(\eta^{2}=.048\right)$, and the between-study variance $\left(\eta^{2}=.032\right)$ had the highest impact on the MSE. Therefore, in Table 3 only the results categorized according to these factors are provided. As expected, a smaller MSE was found when the number of measurement occasions and the number of studies increased, and the between-study variance decreased. In general, applying Hedges' correction leads to better estimates in the same conditions compared to the other approaches, whereas the traditional and constrained variance approaches perform very similar. For example, when $K=10, J=4, I=10, \sigma_{u_{2}}^{2}=2, \sigma_{v_{2}}^{2}=2$, the MSE for the immediate effect was 0.516 for the no adjustment procedure and constraining the level-1 variance to one procedure whereas it was 0.331 for Hedges' correction approach. As the number of studies $(K)$ increased to 30 for the 
same conditions, the MSE decreased to 0.242 for the first two procedures and 0.112 for Hedges' correction approach.

[Table 3 near here]

Estimates of standard errors of the average treatment effects.

An ANOVA revealed that the number of measurement occasions $\left(\eta^{2}=.131\right)$ and the number of studies $\left(\eta^{2}=.278\right)$ had the most substantial impact on the gap between the standard error estimates and the standard deviation of the estimates. To a smaller extent, the number of cases $\left(\eta^{2}=.071\right)$ and the between-study variance $\left(\eta^{2}=.036\right)$ affected the variation in these estimates. As the results show (Table 4), the relative bias of standard errors is almost the same across all approaches. In all conditions, the relative bias was negative, which indicates the underestimation of standard errors across all approaches and conditions. However, none of the bias values exceeded Hoogland and Boomsma's (1998) threshold of .10. The results of the As the number of measurement occasions and the number of studies increases, the bias decreases. All approaches performed similarly in estimating the standard errors of fixed effects. Note that this does not mean that all approaches give the same standard errors. Yet, because Hedges' correction factor was applied to both the effect size and standard error estimates, the standard deviation of the estimates and the mean standard error were affected to the same degree, resulting in the same relative bias for the standard errors.

[Table 4 near here] 


\section{Coverage proportion for the estimated effects}

Another way to evaluate the effects estimates and the corresponding standard error estimates is to check the coverage proportion of the confidence intervals. Due to simulating 2000 datasets for each condition, we expect the coverage proportion to be close to the nominal value of .95 . The standard error of the estimated proportion can be approximated as follows: $S E(p)=$ $\sqrt{p(1-p) / N}$, in which $p$ indicates the probability (i.e., .95$)$, and $N$ stands for the number of replications. This means that, if the coverage proportion is .95 , there is a $95 \%$ probability that by drawing randomly 2000 datasets, the estimated coverage proportion is between .94 and .96 $(=.95 \pm 1.96 * \sqrt{.95 * .05 / 2000})$. Tables 5 and 6 illustrate that when Hedges' correction procedure was applied, the coverage proportions were within this expected range for almost all conditions. For the traditional and constrained variance approaches, the coverage proportion is however too small in most conditions. The coverage proportion of the immediate treatment effect estimates was decreasing when the number of studies increased to 30 . This was especially the case when the number of measurement occasions was 10, and it went down to the problematic value of .68 in some conditions. Similar results were found for the standardized effect sizes.

In contrast, the confidence interval coverage proportion was appropriate for the estimates of treatment effect on time trend across most of the conditions and all approaches. The smallest value of the coverage proportion for the estimation of treatment effect on time trend was not lower than .92 in all conditions. An explanation is that for the estimated effect on trend, a smaller relative bias of standard errors was found.

[Table 5 near here] 
[Table 6 near here]

\section{Variance Components}

We looked at the variance component estimates (i.e., between-case and between-study variances) for both the immediate treatment effect and the effect on trend to evaluate their recovery. Due to constraining the variance component estimates to be nonnegative, the distribution of the estimates is positively skewed. Therefore, to evaluate the relative bias in the estimator, we calculated the median relative deviation of the estimates from the population value instead of mean relative deviation.

The ANOVA showed that that the underlying model $\left(\eta^{2}=.016\right)$ and the number of measurement occasions $\left(\eta^{2}=.016\right)$ slightly affected the between-study variance estimates. The results (Table 7) show that in the standard and the constrained variance procedures, the positive relative bias observed in conditions with smaller measurement occasions $(I=10)$ increased by increasing the number of subjects and the number of studies. When standardized raw data were synthesized using the standard or constrained variance approach, the relative bias decreases significantly as the number of measurement occasions (I) increases from 10 to 20 . When Hedges' bias correction is applied, the between-study variance estimates are already underestimated when the number of measurement occasions is 10 , which results in a slightly more severe underestimation as the number of measurement occasions increases from 10 to 20 across all conditions, although for $K=30$ the estimations are unbiased regardless the other conditions. For the first two approaches (i.e., no adjustment procedure or constraining the level1 variance to one), the condition with maximum relative bias (i.e., 0.342 and 0.354 , respectively) was $K=30, J=7, I=10, \sigma_{u_{2}}^{2}=0.5, \sigma_{v_{2}}^{2}=0.5$. When Hedges' correction procedure 
was applied, the condition with $K=10, J=4, I=20, \sigma_{u_{2}}^{2}=2, \sigma_{v_{2}}^{2}=0.5$ had the maximum relative bias (i.e., -0.157).

Closer inspection of Table 7 reveals that when the number of measurement occasions is smaller, Hedges' correction procedure leads to better between-study variance estimates when applied to standardized raw data compared with standardized effect sizes; in contrast, the constraining the level-1 variance to one results in less bias when fitted to standardized effect sizes. However, for larger measurement occasions, the results are more or less similar in both standardized raw data and standardized effect sizes.

According to the results of ANOVA, the number of measurement occasions $\left(\eta^{2}=\right.$ .171) had the largest effect on the variation of between-case variance estimates. The betweencase variance $\left(\eta^{2}=.090\right)$, the interaction of the number of measurement occasions and the between-case variance $\left(\eta^{2}=.033\right)$, and the underlying model $\left(\eta^{2}=.020\right)$ were other factors that affected these estimates. As can be seen in Table 8, the between-case variance tends to be overestimated significantly, especially for a smaller number of measurement occasions (almost four times larger than the condition with larger measurement occasions). From these results, it can be seen that as the number of studies increases, the relative bias slightly increases. The bias of between-case variance estimates is significantly larger than the bias of the between-study variance estimates. In contrast to the between-study variance, fitting the constraining the level1 variance to one to standardized raw data results in better estimation compared with applying to standardized effect sizes, whereas Hedges' correction procedure has a better performance when applied to standardized effect sizes. Closer inspection reveals that smaller bias is observed across all models and both kind of data analyses when the between-case variance is larger $\left(\sigma_{u_{2}}^{2}=2\right)$. In general, applying Hedges' correction factor performs better over other models and results in less biased estimates when the number of measurement occasions is smaller. 
[Table 7 near here]

[Table 8 near here] 


\section{Discussion}

Previous studies (Moeyaert et al., 2013; Ugille et al., 2012, 2014) evaluating the multilevel meta-analysis of standardized SCED raw data and standardized SCED effect sizes proposed by Van den Noortgate and Onghena (2003a, 2003b, 2008) have shown that the estimates of the treatment effects are substantially biased when the measurement occasions per cases are smaller than 20 . The present study was set up to detect the performance of some alternative approaches in combining standardized SCED data with small measurement occasions (20 or fewer). More specifically, the aim of this study was to assess the performance of two different adjustments (i.e., applying Hedges' bias correction and constraining the level-1 variance) and the default approach with no adjustment, in recovering the fixed effects and variance components when conducting a three-level meta-analysis of both standardized raw data and standardized effect sizes.

The studies of Ugille et al. (2012) and Ugille et al. (2014) indicated that the variance component estimates were biased when synthesizing standardized effect sizes and to a higher extent when combining standardized raw data. These studies further found that the estimates were more inflated when the measurement occasions were 20 or less. Therefore, simulation conditions of the current study were chosen to be similar to the conditions of these studies as described in the method section. The research question was whether and to what degree the results of raw data and effect size analyses could be improved by implementing the two adjustments described above. Standardization is of particular importance when the outcome from different SCED studies is measured on different scales. In such cases, SCED metaanalysts have to standardize the data before performing multilevel meta-analysis. 


\section{Summary of the main findings}

The results of this study show that, in general, Hedges' correction procedure is promising to reduce the bias of the fixed effect estimation for both combining standardized raw data and standardized effect sizes. A similar appealing performance of Hedges' bias correction for synthesizing standardized SCED effect sizes was found in the study of Ugille et al. (2014). The bias of average treatment effect estimate is close to zero across all conditions applying this procedure. For the other procedures, the results consistently show that increasing the number of measurement occasions leads to almost unbiased treatment effects' estimates. We obtained smaller MSE values when Hedges' bias correction applied to synthesizing standardized SCED raw data compared to what was found in the study of Moeyaert et al. (2013) particularly when the number of studies is larger and the number of measurement occasions and the betweenstudy variance are smaller. These results also confirm the promising performance of using Hedges' bias correction to combine the standardized SCED raw data.

Another major finding was that in general the coverage proportion was often too small when only 10 measurement occasions per subject are included. The results are even worse as the number of subjects per study and the number of studies increase. An explanation is that when there are only 10 measurement occasions per subjects, the effect is likely to be underestimated. This is particularly observable in the case of large number of studies, resulting to the smaller standard errors and therefore confidence intervals. The coverage proportions are appropriate for the average treatment effect estimates across all conditions when Hedges' bias correction procedure is applied. Another promising finding compared to what was found in the study of Moeyaert et al. (2013) is the better coverage proportion for the estimated immediate treatment effects when this bias correction is applied to standardized SCED raw data, especially when the number of studies is larger and the number of measurement is smaller. Taken together, these 
results suggest that in the conditions with more studies $(K=30)$ and more measurement occasions $(I=20)$, the average treatment effects are unbiased in all approaches.

Surprisingly, the results are quite different from treatment effect estimates in terms of variation in the treatment effects over case and study levels. The results show for the traditional approach a high overestimation of the between-case variance, and to a smaller degree of the betweenstudy variance, especially when the number of measurement occasions per phase is only 10 . When the number of measurement occasions increases from 10 to 20 , the relative bias of estimates decreases significantly. This study contributes to our understanding of better performance of Hedges' correction in combination with different conditions when the number of measurement occasions is small (20 or fewer). The advantages of Hedges' correction relative over the unadjusted and variance constrained methods are more pronounced when the series length is short $(I=10)$, no matter how many cases or studies are combined. Comparing our results with those in the study of Moeyaert et al. (2013) confirm the impact of applying Hedges' bias correction on decreasing the bias of variance components when combining SCED raw data. Hedges' bias correction performs differently when estimating the variance components compared to the fixed effects, both when combining standardized effect sizes or standardized raw data. Applying Hedges' correction procedure results in an underestimation of the betweenstudy variance estimates across all conditions, but unbiased estimates were observed with more studies $(K=30)$. Interestingly, the bias is worse when combining standardized effect sizes rather than standardized raw data when the number of measurement occasions is 10 . The results are the other way around when estimating the between-case variances. Much higher bias is observed when standardized raw data are combined, particularly for smaller measurement occasions. Due to the fact that Hedges' bias correction is based on the degrees of freedom, which in the SCED meta-analysis depends on the number of measurement occasions, applying this correction factor has greater effect when the measurement occasions are just 10. 


\section{Limitations and future research}

However, these results need to be interpreted with caution due to the inherent limitations of the simulation study. The results can be only generalized to the simulation conditions. First, we simulated our data only for MBDs (which are the most frequently applied SCEDs) and did not include other types of SCED. Second, we did not generate the data with autocorrelation among residuals in our simulation. One possible feature of SCED data that can occur due to the repeated measurements is that the observations closer in time are more likely to be similar compared to farther observations. Ignoring the existing autocorrelation results in biased variance components. Several studies have investigated the consequences of ignoring or modeling autocorrelation in the context of SCEDs (Ferron et al., 2009; Petit-Bois et al., 2016). Although we are aware of the problematic consequences of ignoring, oversimplifying, or misspecifing of this phenomenon, accounting for and modeling the autocorrelation was not the focus of the current study. Therefore, we did not generate the autocorrelated SCED data in our simulation to be further modeled in our analyses.

Moreover, we only considered continuous normally distributed outcomes. Shadish \& Sullivan (2011) surveyed over 800 SCED studies and found that many of outcomes used were count-based. However, most parametric effect sizes and models that are used with these data are based on assumptions that the data are interval- or ratio-scaled and normally distributed, whereas the may require other distributional assumptions. A recent study compared the performance of generalized linear mixed model (GLMM) over linear mixed model (LMM) in analyzing SCED count data through a simulation study (Declercq et al., 2018). The former model can account for discrete nature of SCED count data. The results indicated that GLMM does not substantially outperform LMM. Further studies are required to investigate the complexities of multilevel modeling of SCED count data. 
Finally, we only generated data with linear trajectories and more complex data patterns need consideration in future studies. Modeling and accounting for all complexities in multilevel modeling at once is not feasible and researchers are forced to investigate limited complexities and extend their models in future studies in accordance with their findings. Because multilevel modeling of SCEDs is a recent field of research, there are still a lot to learn about this approach.

We started with a relatively simple model to get a clear view on the influence of these adjustments. Future research could study the approaches in more complex situations, for instance when there is autocorrelation, covariance between regression coefficients, nonlinear trajectories, count or proportion outcomes, a mixture of different SCED designs, or non-normal distributions for the residuals at each level. We do not see reasons, however, why the use of the bias-correction factor would lead to better results in these more complex scenarios.

\section{Conclusions and recommendations}

In general, current results show that applying Hedges' bias correction in combining data from SCEDs with a small number of measurement occasions and a small number of cases per study in multilevel meta-analysis, results in a substantial reduction of bias. We therefore recommend applied researchers to use this bias correction factor because it results to less biased estimates of the treatment effects and the variance components compared to other methods in the current study. We also recommend software developers (e.g., MultiSCED; Declercq et al., 2019) to introduce an option for Hedges' bias correction (as the best performer according to the results of current study) to make it possible for SCED researchers to apply it in their analyses. Although the between-case variance estimates were found to be biased across almost all conditions, applying Hedges' bias correction still performs better than other methods and results in less biased fixed effects and variance components estimates. Our final point to SCED meta-analysts is that in terms of fixed effects estimation, applying Hedges' bias correction performs equally 
appropriate in both synthesizing the standardized SCED effect sizes and the standardized SCED raw data, resulting in unbiased estimates regardless of the kind of data. In terms of variance components, if SCED meta-analysts are interested in the variability between cases, then synthesizing standardized effect sizes applying Hedges' bias correction rather than raw data will result in less biased estimates. However, for the variation between studies, applying this correction to synthesizing raw data will lead to better estimates.

\section{Acknowledgement}

This project was supported in part by the Institute of Education Sciences, U.S. Department of Education, under Grant R305D150007. All the content is solely the responsibility of the authors and do not represent views of the Institute of Education Sciences, U.S. Department of Education. For the simulation, we used equipment at the VSC-Flemish Supercomputer Center, funded by the Hercules Foundation and the Flemish Government, Department EWI.

\section{References}

Allison, D. B., \& Gorman, B. S. (1993). Calculating effect sizes for meta-analysis: The case of the single case. Behaviour Research and Therapy, 31(6), 621-631. https://doi.org/10.1016/0005-7967(93)90115-B

Barlow, D. H., Nock, M. K., \& Hersen, M. (2009). Single case experimental designs: Strategies for studying behavior change (3rd ed.). Boston: Pearson/Allyn and Bacon.

Center, B. A., Skiba, R. J., \& Casey, A. (1985). A methodology for the quantitative synthesis of intra-subject design research. The Journal of Special Education, 19(4), 387-400. https://doi.org/10.1177\%2F002246698501900404

Cohen, J. (1988). Statistical power analysis for the behavioral sciences (2nd ed.). Hillsdale, 
NJ: Lawrence Erlbaum Associates Publishers.

Declercq, L., Cools, W., Beretvas, S. N., Moeyaert, M., Ferron, J. M., \& Van den Noortgate, W. (2019). MultiSCED: A tool for (meta-)analyzing single-case experimental data with multilevel modeling. Behavior Research Methods. https://doi.org/10.3758/s13428-019$01216-2$

Fan, X., Felsovalyi, A., Sivo, S. A., \& Keenan, S. C. (2002). SAS® for Monte Carlo studies: A guide for quantitative researchers. Cary, NC: SAS Institute Inc.

Ferron, J. M., Bell, B. A., Hess, M. R., Rendina-Gobioff, G., \& Hibbard, S. T. (2009). Making treatment effect inferences from multiple-baseline data: The utility of multilevel modeling approaches. Behavior Research Methods, 41(2), 372-384. https://doi.org/10.3758/BRM.41.2.372

Hedges, L. V. (1981). Distribution theory for Glass 's estimator of effect size and related estimators. Journal of Educational Statistics, 6(2), 107-128. https://doi.org/10.2307/1164588

Hedges, L. V, Pustejovsky, J. E., \& Shadish, W. R. (2012). A standardized mean difference effect size for single case designs. Research Synthesis Methodsynthesis Methods, 3(3), 224-239. https://doi.org/10.1002/jrsm.1052

Hedges, L. V, Pustejovsky, J. E., \& Shadish, W. R. (2013). A standardized mean difference effect size for multiple baseline designs across individuals. Research Synthesis Methods, 4(4), 324-341. https://doi.org/10.1002/jrsm.1086

Hembry, I., Bunuan, R., Beretvas, S. N., Ferron, J. M., \& Van den Noortgate, W. (2015). Estimation of a nonlinear intervention phase trajectory for multiple-baseline design data. The Journal of Experimental Education, 83(4), 514-546.

https://doi.org/10.1080/00220973.2014.907231

Hoogland, J. J., \& Boomsma, A. (1998). Robustness studies in covariance structure modeling: 
An overview and a meta-analysis. Sociological Methods \& Research, 26(3), 329-367. https://doi.org/10.1177/0049124198026003003

Horner, R. H., Carr, E. G., Halle, J., McGee, G., Odom, S. L., \& Wolery, M. (2005). The use of single-subject research to identify evidence-based practice in special education. Exceptional Children, 71(2), 165-179. https://doi.org/10.1177/001440290507100203

Horner, R. H., \& Odom, S. L. (2014). Constructing single-case research designs: Logic and options. In T. R. Kratochwill \& J. R. Levin (Eds.), Single-case intervention research: methodological and statistical advances (pp. 27-51). Washington: American Psychological Association.

Hox, J. J., \& de Leeuw, E. D. (2003). Multilevel models for meta-analysis. In S. P. Reise \& N. Duan (Eds.), multilevel modeling: Methodological advances, issues, and applications (pp. 90-111). Mahwah, NJ: Lawrence Erlbaum Associates Publishers.

Kratochwill, T. R., Hitchcock, J. H., Horner, R. H., Levin, J. R., Odom, S. L., Rindskopf, D. M., \& Shadish, W. R. (2010). Single-case designs technical documentation. Retrieved from http://ies.ed.gov/ncee/wwc/pdf/wwc_scd.pdf

Kratochwill, T. R., \& Levin, J. R. (2014). Introduction: An overview of single-case intervention research. In T. R. Kratochwill \& J. R. Levin (Eds.), Single-case intervention research: Methodological and statistical advances (pp. 3-23). Washington: American Psychological Association.

Moeyaert, M., Ugille, M., Ferron, J. M., Beretvas, S. N., \& Van den Noortgate, W. (2013). The three-level synthesis of standardized single-subject experimental data: A monte carlo simulation study. Multivariate Behavioral Research, 48(5), 719-748. https://doi.org/10.1080/00273171.2013.816621

Moeyaert, M., Ugille, M., Ferron, J. M., Beretvas, S. N., \& Van den Noortgate, W. (2014). Three-level analysis of single-case experimental data: Empirical validation. The Journal 
of Experimental Education, 82(1), 1-21. https://doi.org/10.1080/00220973.2012.745470

Morgan, D. L., \& Morgan, R. K. (2001). Single-participant research design: Bringing science to managed care. The American Psychologist, 56(2), 119-127.

https://doi.org/10.1037//0003-066X.56.2.119

Onghena, P. (2005). Single-case designs. In B. S. Everittt \& D. C. Howell (Eds.), Encyclopedia of statistics in behavioral science (pp. 1850-1854). Chichester: John Wiley \& Sons.

Owens, C. M. (2011). Meta-analysis of single-case data: A monte carlo investigation of a three level model. Graduate Thesis and Dissertation. Retrieved from http://scholarcommons.usf.edu/etd/3273

Parker, R. I., Vannest, K. J., \& Davis, J. L. (2014). Non-overlap analysis of shigle-case research. In T. R. Kratochwill \& J. R. Levin (Eds.), Single-case intervention research: methodological and statistical advances (pp. 127-152). Washington: American Psychological Association.

Petit-Bois, M., Baek, E., Van den Noortgate, W., Beretvas, S. N., \& Ferron, J. M. (2016). The consequences of modeling autocorrelation when synthesizing single-case studies using a three-level model. Behavior Research Methods, 48(2), 803-812. https://doi.org/10.3758/s13428-015-0612-1

Riley-Tillman, T. C. hris, \& Burns, M. K. (2009). Evaluating educational interventions: Single-case design for measuring response to intervention. New York: The Guilford Press.

SAS Institute, I. (2017). Base SAS® 9.4 procedures guide (7th ed.). Cary, NC: SAS Institute Inc.

Shadish, W. R. (2014). Statistical analyses of single-case designs : The shape of things to come. Current Directions in Psychological Science, 23(2), 139-146. 
https://doi.org/10.1177/0963721414524773

Shadish, W. R., Hedges, L. V, Horner, R. H., \& Odom, S. L. (2015). The role of between-case effect size in conducting, interpreting, and summarizing single-case research(NCER 2015-002). National Center for Education Research, Institute of Education. Retrieved from http://ies.ed.gov/ncser/pubs/2015002/

Shadish, W. R., \& Sullivan, K. J. (2011). Characteristics of single-case designs used to assess intervention effects in 2008. Behavior Research Methods, 43, 971-980. https://doi.org/10.3758/s13428-011-0111-y

Ugille, M., Moeyaert, M., Beretvas, S. N., Ferron, J. M., \& Van den Noortgate, W. (2012). Multilevel meta-analysis of single-subject experimental designs : A simulation study. Behavior Research Methods, 44(4), 1244-1254. https://doi.org/10.3758/s13428-0120213-1

Ugille, M., Moeyaert, M., Beretvas, S. N., Ferron, J. M., \& Van den Noortgate, W. (2014). Bias corrections for standardized effect size estimates used with single-subject experimental designs. The Journal of Experimental Education, 82(3), 358-374. https://doi.org/10.1080/00220973.2013.813366

Van den Noortgate, W., \& Onghena, P. (2003a). Combining single-case experimental data using hierarchical linear models. School Psychology Quarterly, 18(3), 325-346. https://doi.org/10.1521/scpq.18.3.325.22577

Van den Noortgate, W., \& Onghena, P. (2003b). Hierarchical linear models for the quantitative integration of effect sizes in single-case research. Behavior Research Methods, Instruments, \& Computers, 35(1), 1-10. https://doi.org/10.3758/BF03195492 Van den Noortgate, W., \& Onghena, P. (2008). A multilevel meta-analysis of single-subject experimental design studies. Evidence-Based Communication Assessment and Intervention, 2(3), 142-151. https://doi.org/10.1080/17489530802505362 
Table 1. Timing of intervention introduction.

\begin{tabular}{ccc}
\hline \multirow{2}{*}{$\begin{array}{c}\text { Number of measurement } \\
\text { occasions }(I)\end{array}$} & \multicolumn{2}{c}{ Number of cases $(J)$} \\
\cline { 2 - 3 } & 4 & 7 \\
\hline 10 & $4,5,7,8$ & $4,5,5,6,7,7,8$ \\
20 & $7,10,12,15$ & $7,9,9,11,13,13,15$ \\
\hline
\end{tabular}


Table 2. Mean and variance of relative bias for the estimated immediate effect $\left(\hat{\gamma}_{200}\right)$.

\begin{tabular}{|c|c|c|c|c|c|c|c|c|}
\hline \multirow{3}{*}{ Model } & \multicolumn{4}{|c|}{ Standardized raw data } & \multicolumn{4}{|c|}{ Standardized effect sizes } \\
\hline & \multicolumn{2}{|c|}{ Mean } & \multicolumn{2}{|c|}{ Variance } & \multicolumn{2}{|c|}{ Mean } & \multicolumn{2}{|c|}{ Variance } \\
\hline & $I=10$ & $I=20$ & $I=10$ & $I=20$ & $I=10$ & $I=20$ & $I=10$ & $I=20$ \\
\hline Unadjusted & 0.149 & 0.050 & 0.045 & 0.032 & & & & \\
\hline Constraining level-1 variance to one & 0.149 & 0.050 & 0.045 & 0.032 & 0.149 & 0.050 & 0.046 & 0.032 \\
\hline Hedges' correction & -0.001 & 0.000 & 0.034 & 0.029 & -0.000 & 0.000 & 0.035 & 0.029 \\
\hline
\end{tabular}

Notes. $I=$ number of measurement occasions.

No values are given for the unadjusted model for standardized effect sizes, because by default in synthesizing effect sizes, the level-1 variance is constrained to the estimated sampling variance. 
Table 3. Mean squared error (MSE) of the estimated immediate effect $\left(\hat{\gamma}_{200}\right)$.

\begin{tabular}{|c|c|c|c|c|c|c|c|c|c|}
\hline \multirow{3}{*}{ Model } & \multicolumn{5}{|c|}{ Standardized raw data } & \multicolumn{4}{|c|}{ Standardized effect sizes } \\
\hline & \multirow[t]{2}{*}{$\sigma_{v_{2}}^{2}$} & \multicolumn{2}{|c|}{$K=10$} & \multicolumn{2}{|c|}{$K=30$} & \multicolumn{2}{|c|}{$K=10$} & \multicolumn{2}{|c|}{$K=30$} \\
\hline & & $I=10$ & $I=20$ & $I=10$ & $I=20$ & $I=10$ & $I=20$ & $I=10$ & $I=20$ \\
\hline \multirow[t]{2}{*}{ Unadjusted } & 0.5 & 0.256 & 0.114 & 0.148 & 0.045 & & & & \\
\hline & 2 & 0.451 & 0.287 & 0.215 & 0.101 & & & & \\
\hline \multirow{2}{*}{$\begin{array}{l}\text { Constraining level-1 } \\
\text { variance to one }\end{array}$} & 0.5 & 0.257 & 0.114 & 0.148 & 0.045 & 0.267 & 0.116 & 0.151 & 0.046 \\
\hline & 2 & 0.451 & 0.287 & 0.215 & 0.101 & 0.460 & 0.288 & 0.219 & 0.101 \\
\hline \multirow[t]{2}{*}{ Hedges' correction } & 0.5 & 0.126 & 0.095 & 0.043 & 0.032 & 0.134 & 0.097 & 0.046 & 0.032 \\
\hline & 2 & 0.277 & 0.251 & 0.094 & 0.082 & 0.284 & 0.251 & 0.097 & 0.083 \\
\hline
\end{tabular}

Notes. $I=$ number of measurement occasions; $K=$ number of studies; $\sigma_{v_{2}}^{2}=$ between-study variance.

No values are given for the unadjusted model for standardized effect sizes, because by default in synthesizing effect sizes, the level-1 variance is constrained to the estimated sampling variance. 
Table 4. Relative difference between the median of the standard error estimates and the standard deviation of the estimated effects.

\begin{tabular}{|c|c|c|c|c|c|c|c|c|}
\hline \multirow{3}{*}{ Model } & \multicolumn{4}{|c|}{ Standardized raw data } & \multicolumn{4}{|c|}{ Standardized effect sizes } \\
\hline & \multicolumn{2}{|c|}{$K=10$} & \multicolumn{2}{|c|}{$K=30$} & \multicolumn{2}{|c|}{$K=10$} & \multicolumn{2}{|c|}{$K=30$} \\
\hline & $I=10$ & $I=20$ & $I=10$ & $I=20$ & $I=10$ & $I=20$ & $I=10$ & $I=20$ \\
\hline Unadjusted & -0.049 & -0.043 & -0.036 & -0.012 & & & & \\
\hline $\begin{array}{l}\text { Constraining level-1 } \\
\text { variance to one }\end{array}$ & -0.049 & -0.043 & -0.036 & -0.012 & -0.042 & -0.040 & -0.030 & -0.013 \\
\hline Hedges' correction & -0.049 & -0.043 & -0.036 & -0.012 & -0.038 & -0.041 & -0.030 & -0.013 \\
\hline
\end{tabular}

Notes. $I=$ number of measurement occasions; $K=$ number of studies.

No values are given for the unadjusted model for standardized effect sizes, because by default in synthesizing effect sizes, the level-1 variance is constrained to the estimated sampling variance. 
Table 5. Coverage proportion for the estimated immediate effect for standardized raw data.

\begin{tabular}{|c|c|c|c|c|c|c|c|c|c|c|}
\hline \multirow[b]{3}{*}{ Model } & \multirow[b]{3}{*}{$\sigma_{u_{2}}^{2}$} & \multirow[b]{3}{*}{$\sigma_{v_{2}}^{2}$} & \multicolumn{4}{|c|}{$K=10$} & \multicolumn{4}{|c|}{$K=30$} \\
\hline & & & \multicolumn{2}{|c|}{$J=4$} & \multicolumn{2}{|c|}{$J=7$} & \multicolumn{2}{|c|}{$J=4$} & \multicolumn{2}{|c|}{$J=7$} \\
\hline & & & $I=10$ & $I=20$ & $I=10$ & $I=20$ & $I=10$ & $I=20$ & $I=10$ & $I=20$ \\
\hline \multirow[t]{4}{*}{ Unadjusted } & 0.5 & 0.5 & .9025 & .9380 & .8910 & .9365 & .7700 & .9185 & .6835 & .9065 \\
\hline & & 2 & .9300 & .9450 & .9305 & .9370 & .8775 & .9355 & .8660 & .9400 \\
\hline & 2 & 0.5 & .9275 & .9560 & .9085 & .9430 & .8205 & .9255 & .7435 & .9130 \\
\hline & & 2 & .9365 & .9460 & .9420 & .9425 & .8880 & .9445 & .8690 & .9315 \\
\hline \multirow{4}{*}{$\begin{array}{l}\text { Constraining level-1 } \\
\text { variance to one }\end{array}$} & 0.5 & 0.5 & .9015 & .9385 & .8915 & .9365 & .7700 & .9185 & .6830 & .9065 \\
\hline & & 2 & .9305 & .9450 & .9300 & .9370 & .8780 & .9355 & .8655 & .9400 \\
\hline & 2 & 0.5 & .9275 & .9560 & .9075 & .9430 & .8205 & .9255 & .7435 & .9130 \\
\hline & & 2 & .9365 & .9460 & .9425 & .9425 & .8885 & .9445 & .8685 & .9315 \\
\hline \multirow[t]{4}{*}{ Hedges' correction } & 0.5 & 0.5 & .9505 & .9490 & .9515 & .9545 & .9390 & .9570 & .9490 & .9405 \\
\hline & & 2 & .9465 & .9480 & .9415 & .9420 & .9535 & .9450 & .9435 & .9485 \\
\hline & 2 & 0.5 & .9520 & .9540 & .9510 & .9495 & .9400 & .9465 & .9410 & .9495 \\
\hline & & 2 & .9470 & .9485 & .9570 & .9515 & .9510 & .9485 & .9460 & .9490 \\
\hline
\end{tabular}

Notes. $I=$ number of measurement occasions; $J=$ number of cases; $K=$ number of studies; $\sigma_{u_{2}}^{2}=$ between-case variance; $\sigma_{v_{2}}^{2}=$ between-study variance;

Coverage proportions between .94 and .96 are in boldface. 
Table 6. Coverage proportion for the estimated immediate effect for standardized effect sizes.

\begin{tabular}{|c|c|c|c|c|c|c|c|c|c|c|}
\hline \multirow[b]{3}{*}{ Model } & \multirow[b]{3}{*}{$\sigma_{u_{2}}^{2}$} & \multirow[b]{3}{*}{$\sigma_{v_{2}}^{2}$} & \multicolumn{4}{|c|}{$K=10$} & \multicolumn{4}{|c|}{$K=30$} \\
\hline & & & \multicolumn{2}{|c|}{$J=4$} & \multicolumn{2}{|c|}{$J=7$} & \multicolumn{2}{|c|}{$J=4$} & \multicolumn{2}{|c|}{$J=7$} \\
\hline & & & $I=10$ & $I=20$ & $I=10$ & $I=20$ & $I=10$ & $I=20$ & $I=10$ & $I=20$ \\
\hline \multirow{4}{*}{$\begin{array}{l}\text { Constraining level-1 } \\
\text { variance to one }\end{array}$} & 0.5 & 0.5 & .9160 & .9370 & .9045 & .9370 & .7930 & .9230 & .7030 & .9080 \\
\hline & & 2 & .9250 & .9465 & .9300 & .9365 & .8795 & .9365 & .8675 & .9385 \\
\hline & 2 & 0.5 & .9280 & .9535 & .9185 & .9435 & .8320 & .9270 & .7530 & .9155 \\
\hline & & 2 & .9355 & .9455 & .9385 & .9460 & .8890 & .9425 & .8675 & .9330 \\
\hline \multirow[t]{4}{*}{ Hedges' correction } & 0.5 & 0.5 & .9640 & .9505 & .9585 & .9495 & .9410 & .9565 & .9515 & .9430 \\
\hline & & 2 & .9530 & .9485 & .9450 & .9440 & .9540 & .9450 & .9445 & .9495 \\
\hline & 2 & 0.5 & .9545 & .9560 & .9575 & .9500 & .9445 & .9480 & .9430 & .9480 \\
\hline & & 2 & .9475 & .9460 & .9560 & .9520 & .9460 & .9500 & .9480 & .9470 \\
\hline
\end{tabular}

Notes. $I=$ number of measurement occasions; $J=$ number of cases; $K=$ number of studies; $\sigma_{u_{2}}^{2}=$ between-case variance; $\sigma_{v_{2}}^{2}=$ between-study variance;

Coverage proportions between .94 and .96 are in boldface 
Table 7. Median of relative deviation of the between-study variance estimates of $\gamma_{200}$.

\begin{tabular}{|c|c|c|c|c|c|c|c|c|c|c|c|c|c|c|c|c|c|c|}
\hline \multirow[b]{4}{*}{ Model } & \multirow[b]{4}{*}{$\sigma_{u_{2}}^{2}$} & \multirow[b]{4}{*}{$\sigma_{v_{2}}^{2}$} & \multicolumn{8}{|c|}{ Standardized raw data } & \multicolumn{8}{|c|}{ Standardized effect size } \\
\hline & & & \multicolumn{4}{|c|}{$K=10$} & \multicolumn{4}{|c|}{$K=30$} & \multicolumn{4}{|c|}{$K=10$} & \multicolumn{4}{|c|}{$K=30$} \\
\hline & & & \multicolumn{2}{|c|}{$J=4$} & \multicolumn{2}{|c|}{$J=7$} & \multicolumn{2}{|c|}{$J=4$} & \multicolumn{2}{|c|}{$J=7$} & \multicolumn{2}{|c|}{$J=4$} & \multicolumn{2}{|c|}{$J=7$} & \multicolumn{2}{|c|}{$J=4$} & \multicolumn{2}{|c|}{$J=7$} \\
\hline & & & $I=10$ & $I=20$ & $I=10$ & $I=20$ & $I=10$ & $I=20$ & $I=10$ & $I=20$ & $I=10$ & $I=20$ & $I=10$ & $I=20$ & $I=10$ & $I=20$ & $I=10$ & $I=20$ \\
\hline \multirow[t]{4}{*}{ Unadjusted } & 0.5 & 0.5 & 0.184 & -0.057 & 0.213 & -0.009 & 0.313 & 0.052 & 0.342 & 0.052 & & & & & & & & \\
\hline & & 2 & 0.186 & -0.017 & 0.250 & 0.026 & 0.301 & 0.076 & 0.298 & 0.092 & & & & & & & & \\
\hline & 2 & 0.5 & 0.123 & -0.070 & 0.146 & -0.061 & 0.232 & 0.075 & 0.280 & 0.055 & & & & & & & & \\
\hline & & 2 & 0.178 & -0.002 & 0.193 & 0.031 & 0.307 & 0.076 & 0.288 & 0.063 & & & & & & & & \\
\hline \multirow{4}{*}{$\begin{array}{l}\text { Constraining } \\
\text { level-1 variance }\end{array}$} & 0.5 & 0.5 & 0.194 & -0.057 & 0.224 & -0.009 & 0.323 & 0.053 & 0.354 & 0.053 & 0.057 & -0.058 & 0.159 & -0.014 & 0.219 & 0.026 & 0.295 & 0.051 \\
\hline & & 2 & 0.191 & -0.018 & 0.252 & 0.027 & 0.304 & 0.076 & 0.299 & 0.092 & 0.161 & -0.022 & 0.226 & 0.026 & 0.281 & 0.067 & 0.270 & 0.092 \\
\hline & 2 & 0.5 & 0.118 & -0.069 & 0.148 & -0.061 & 0.235 & 0.076 & 0.282 & 0.056 & 0.075 & -0.074 & 0.100 & -0.068 & 0.221 & 0.082 & 0.249 & 0.057 \\
\hline & & 2 & 0.177 & -0.002 & 0.194 & 0.030 & 0.308 & 0.076 & 0.289 & 0.062 & 0.147 & -0.002 & 0.170 & 0.032 & 0.278 & 0.077 & 0.278 & 0.065 \\
\hline \multirow{4}{*}{$\begin{array}{l}\text { Hedges' } \\
\text { correction }\end{array}$} & 0.5 & 0.5 & -0.105 & -0.144 & -0.083 & -0.101 & -0.007 & -0.045 & 0.015 & -0.045 & -0.285 & -0.143 & -0.142 & -0.106 & $-\mathbf{- 0 . 0 8 9}$ & -0.067 & -0.022 & -0.047 \\
\hline & & 2 & -0.103 & -0.109 & -0.055 & -0.069 & -0.016 & -0.024 & -0.018 & -0.010 & -0.140 & -0.113 & -0.075 & -0.070 & $-\mathbf{- 0 . 0 3 0}$ & -0.032 & -0.040 & -0.009 \\
\hline & 2 & 0.5 & -0.151 & -0.157 & -0.133 & -0.148 & -0.069 & -0.025 & -0.032 & -0.043 & -0.197 & -0.160 & -0.168 & -0.153 & -0.082 & -0.017 & -0.056 & -0.041 \\
\hline & & 2 & -0.109 & -0.095 & -0.098 & -0.065 & -0.012 & -0.024 & -0.026 & -0.036 & -0.134 & -0.094 & -0.112 & -0.064 & $-\mathbf{- 0 . 0 3 3}$ & -0.024 & $-\mathbf{0 . 0 3 4}$ & -0.035 \\
\hline
\end{tabular}

Notes. $I=$ number of measurement occasions; $J=$ number of cases; $K=$ number of studies; $\sigma_{u_{2}}^{2}=$ between-case variance; $\sigma_{v_{2}}^{2}=$ between-study variance.

a) The conditions with unbiased estimates (less than .10 according to Hoogland \& Boomsma's cutoff) are in boldface; b) No values are given for the unadjusted model for standardized effect sizes, because by default in synthesizing effect sizes, the level-1 variance is constrained to the estimated sampling variance. 
Table 8. Median of relative deviation of the between-case variance estimates of $\gamma_{200}$.

\begin{tabular}{|c|c|c|c|c|c|c|c|c|c|c|c|c|c|c|c|c|c|c|}
\hline \multirow[b]{4}{*}{ Model } & \multirow[b]{4}{*}{$\sigma_{u_{2}}^{2}$} & \multirow[b]{4}{*}{$\sigma_{v_{2}}^{2}$} & \multicolumn{8}{|c|}{ Standardized raw data } & \multicolumn{8}{|c|}{ Standardized effect size } \\
\hline & & & \multicolumn{4}{|c|}{$K=10$} & \multicolumn{4}{|c|}{$K=30$} & \multicolumn{4}{|c|}{$K=10$} & \multicolumn{4}{|c|}{$K=30$} \\
\hline & & & \multicolumn{2}{|c|}{$J=4$} & \multicolumn{2}{|c|}{$J=7$} & \multicolumn{2}{|c|}{$J=4$} & \multicolumn{2}{|c|}{$J=7$} & \multicolumn{2}{|c|}{$J=4$} & \multicolumn{2}{|c|}{$J=7$} & \multicolumn{2}{|c|}{$J=4$} & \multicolumn{2}{|c|}{$J=7$} \\
\hline & & & $I=10$ & $I=20$ & $I=10$ & $I=20$ & $I=10$ & $I=20$ & $I=10$ & $I=20$ & $I=10$ & $I=20$ & $I=10$ & $I=20$ & $I=10$ & $I=20$ & $\mathrm{I}=10$ & $\mathrm{I}=20$ \\
\hline \multirow[t]{4}{*}{ Unadjusted } & 0.5 & 0.5 & 2.138 & 0.531 & 2.203 & 0.591 & 2.314 & 0.616 & 2.479 & 0.621 & & & & & & & & \\
\hline & & 2 & 2.464 & 0.631 & 2.636 & 0.674 & 2.804 & 0.700 & 2.915 & 0.717 & & & & & & & & \\
\hline & 2 & 0.5 & 0.854 & 0.204 & 0.980 & 0.231 & 1.001 & 0.249 & 1.052 & 0.274 & & & & & & & & \\
\hline & & 2 & 0.972 & 0.241 & 1.115 & 0.273 & 1.136 & 0.286 & 1.166 & 0.311 & & & & & & & & \\
\hline \multirow{4}{*}{$\begin{array}{l}\text { Constraining } \\
\text { level-1 variance } \\
\text { to one }\end{array}$} & 0.5 & 0.5 & 2.211 & 0.535 & 2.284 & 0.595 & 2.370 & 0.621 & 2.545 & 0.623 & 3.155 & 0.641 & 3.343 & 0.669 & 3.553 & 0.722 & 3.647 & 0.717 \\
\hline & & 2 & 2.539 & 0.633 & 2.696 & 0.679 & 2.860 & 0.704 & 2.971 & 0.718 & 3.569 & 0.744 & 3.857 & 0.756 & 4.022 & 0.813 & 4.135 & 0.831 \\
\hline & 2 & 0.5 & 0.863 & 0.204 & 0.998 & 0.232 & 1.016 & 0.249 & 1.066 & 0.274 & 1.034 & 0.217 & 1.207 & 0.254 & 1.226 & 0.269 & 1.291 & 0.291 \\
\hline & & 2 & 0.995 & 0.242 & 1.129 & 0.273 & 1.154 & 0.286 & 1.180 & 0.311 & 1.205 & 0.257 & 1.325 & 0.294 & 1.402 & 0.299 & 1.387 & 0.325 \\
\hline \multirow{4}{*}{$\begin{array}{l}\text { Hedges' } \\
\text { correction }\end{array}$} & 0.5 & 0.5 & 1.373 & 0.388 & 1.422 & 0.443 & 1.506 & 0.466 & 1.630 & 0.470 & 0.055 & 0.136 & 0.213 & 0.175 & 0.309 & 0.215 & 0.458 & 0.216 \\
\hline & & 2 & 1.619 & 0.479 & 1.749 & 0.519 & 1.876 & 0.542 & 1.961 & 0.557 & 0.291 & 0.238 & 0.630 & 0.254 & 0.662 & 0.299 & 0.816 & 0.322 \\
\hline & 2 & 0.5 & 0.402 & 0.092 & 0.497 & 0.117 & 0.513 & 0.133 & 0.552 & 0.156 & -0.002 & 0.018 & 0.145 & 0.053 & 0.151 & 0.065 & 0.213 & 0.086 \\
\hline & & 2 & 0.491 & 0.126 & 0.599 & 0.155 & 0.615 & 0.166 & 0.638 & 0.189 & 0.117 & 0.054 & 0.232 & 0.087 & 0.274 & 0.092 & 0.284 & 0.116 \\
\hline
\end{tabular}

Notes. $I=$ number of measurement occasions; $J=$ number of cases; $K=$ number of studies; $\sigma_{u_{2}}^{2}=$ between-case variance; $\sigma_{v_{2}}^{2}=$ between-study variance.

No values are given for the unadjusted model for standardized effect sizes, because by default in synthesizing effect sizes, the level-1 variance is constrained to the estimated sampling variance. 


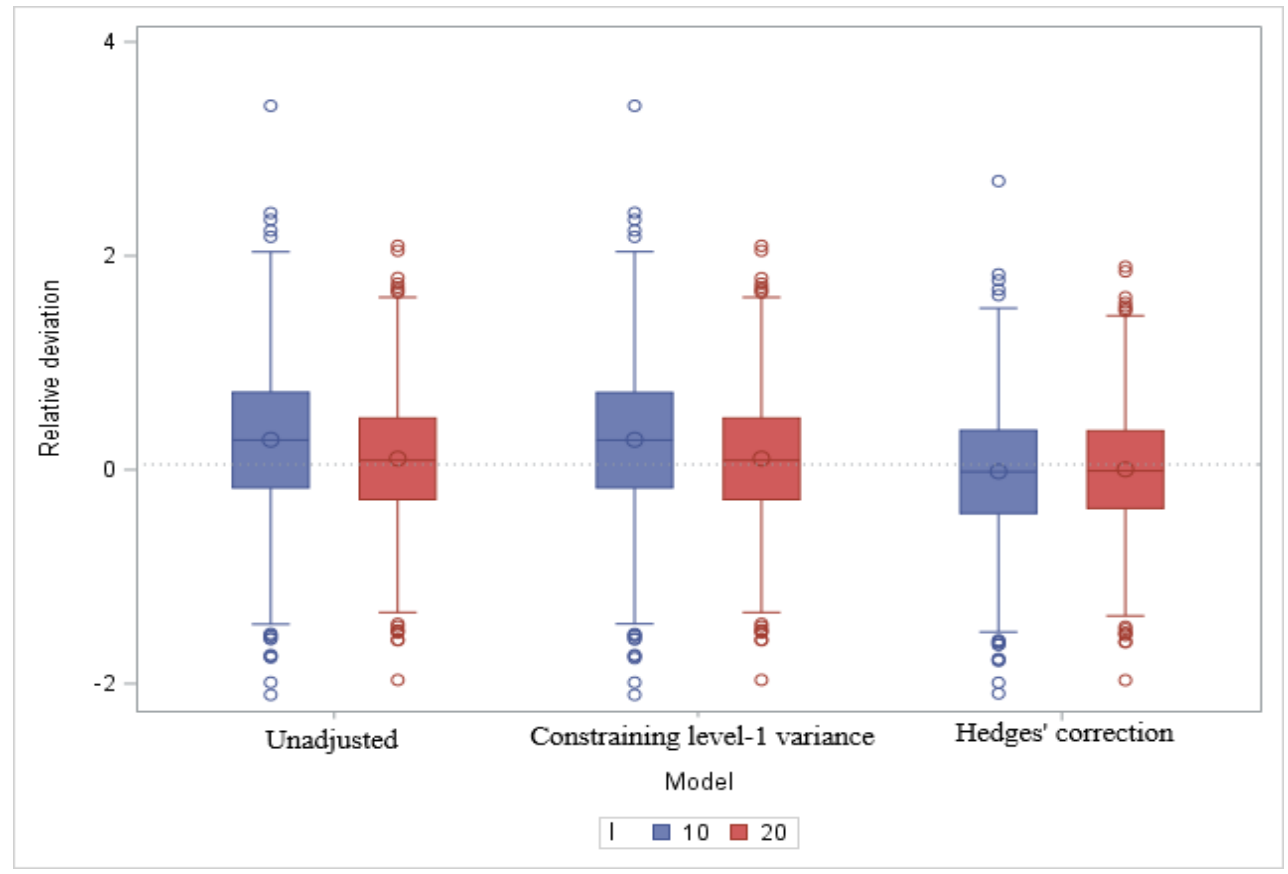

Figure 1. Distribution of the relative deviations of the estimated immediate effect from its populations value $\left(\hat{\gamma}_{200}\right)$, for $K=10, J=4, \sigma_{u_{2}}^{2}=2, \sigma_{v_{2}}^{2}=2$ conditions, with .05 cutoff criterion horizontal line (Hoogland \& Boomsma, 1998). 